\title{
The curse of the black box
}

\author{
Roeland Cortois • Gerlinde Barbara De Deyn
}

Received: 21 June 2011 / Accepted: 12 August 2011 / Published online: 10 September 2011

(C) The Author(s) 2011. This article is published with open access at Springerlink.com

\begin{abstract}
Background Soil is a foremost provider of (agro-) ecosystem services, making plant-soil interactions pivotal in agriculture research. The functioning of soils entails complex interactions between soil biota and the abiotic soil environment and is therefore often considered as a 'black box'. The study of Verbruggen et al. (this volume) tries to crack the black box open by examining the role of soil microbial communities from conventional and organic farming fields for the growth of Zea mays and phosphorous retention in the soil.

Scope In this commentary on the paper of Verbruggen et al. (2011) we use the study to illustrate that investigating soils, and specifically the role of soil biota in ecosystem functioning, is not straightforward, given the overwhelming soil biodiversity and the complexity of soil as a habitat. We discuss the key elements that need to be considered in order to translate results of highly controlled experiments with inoculated soil biota to their functioning in the field.
\end{abstract}

Responsible Editor: Hans Lambers.

R. Cortois $(\triangle) \cdot$ G. B. De Deyn

Department of Terrestrial Ecology,

Netherlands Institute of Ecology,

P.O. Box 50, 6700 AB Wageningen, The Netherlands e-mail: r.cortois@nioo.knaw.nl

G. B. De Deyn

Department of Soil Quality, Wageningen University,

P.O. Box 47, 6700 AA Wageningen, The Netherlands
Conclusions Verbruggen et al. contribute to our understanding of the functional role of AMF in agro-ecosystems. Yet the results only allow us to merely speculate about the realized functional role of AMF communities in the field, a very interesting avenue for future research.

Keywords Inoculation experiment - Soil legacy · Ecosystem functions $\cdot$ AMF $\cdot$ Rhizosphere $\cdot$ Plant-soil interacions

In a world where organic agricultural practice is put forward as a sustainable alternative to conventional farming, understanding the implications of these two contrasting management types for the provision of essential soil functions is more than welcome (Gomiero et al. 2011). From both natural and human dominated ecosystems, it is well known that soil biota play an integral role in soil processes that are essential for sustaining plant productivity and other soil based ecosystem functions (Brussaard et al. 1997). Soil biota are the main drivers of mineral nutrient cycling (Coleman et al. 2004) and also play an important role in causing and suppressing pathogenesis in plants (Garbeva et al. 2004), in the formation of soil structure and in the sequestration of soil carbon (Bronick and Lal 2005; De Deyn et al. 2008). The activity of micro-organisms, meso- and macrofauna in the soil thus affects both abiotic and biological soil properties which are important for plant growth. 
Particularly interesting and relevant for plant performance are the ubiquitous micro-organisms present in the rhizosphere, i.e. the soil environment in the immediate surroundings of the roots. A complex interplay of antagonistic and mutualistic interactions between plants and rhizosphere organisms can give rise to large variations in plant performance resulting from the combined effect of these plant-soil biological interactions (Garbeva et al. 2004; Buée et al. 2009). Antagonistic organisms in the rhizosphere include parasitic nematodes, pathogenic fungi and bacteria (Jackson and Taylor 1996). Among the mutualistic micro-organisms associated with plant roots, arbuscular mycorrhizal fungi (AMF) are the best known. These fungi live partly in plant roots and partly in the soil matrix and provide their host plant with nutrients, mainly phosphorous, in exchange for carbon compounds of the host plant. The effects of AM fungi on plant performance are however not fixed and can be dependent on the specific combination of AMF and plant species (Klironomos 2003), on interactions between AMF and the surrounding soil dwelling organisms (e.g. Azcón-Aguilar and Barea 1996; Barea et al. 1996; Vazquez et al. 2000) and —importantly_on the abiotic soil context in which the plant-AMF interactions take place (Hoeksema et al. 2010). For AMF but also for other groups of soil organisms, especially those which directly interact with plant roots, it has been shown that their effects on plant performance interact with the abiotic properties of the soil, with often stronger impacts of soil biota in soils with lower availability of mineral nutrients (De Deyn et al. 2004; Hoeksema et al. 2010).

Sustainable farming relies on internal biological processes which maintain soil fertility and crop protection under low external input of fertilizers and chemical pest management. A main challenge is thus to identify and protect the functional components of soil biodiversity that provide these ecosystem services (Altieri 1999; Kuyper and Giller 2011). A full understanding of biological soil functioning and the associated ecosystem services under different agricultural management types can, however, only be obtained by taking an integrated perspective, considering short-term as well as long-term interactions between soil biota, abiotic soil properties and plant performance (Barrios 2007).

Studying soils and specifically the role of soil biota in ecosystem functioning is not straightfor- ward, given the overwhelming diversity of soil biota and the opaqueness of soil as a habitat. Indeed, it may not be a surprise that soils are often considered as a 'black box'. Studies on the relation between soil biota and ecosystem functioning are facing the difficulty of choosing between a holistic or reductionist approach. In the holistic approach, a high relevance to natural systems is attained by using natural, complex, soil communities and testing their combined impact on ecosystem response variables, but without knowing the exact underlying mechanisms in play, so that the soil system remains a 'black box'. The reductionist approach on the other hand provides mechanistic insights in soil functioning by focusing on the mechanism by which a specific species or specific group of soil biota affects ecosystem processes in isolation. In this commentary, we discuss the advantages and the pitfalls hidden in both the holistic and reductionist approach using the paper by Verbruggen et al. (2011) (this volume) as a case study, where elements of both approaches have been combined.

The study of Verbruggen et al. (2011) aimed to explore potential differences in the functioning of soil microbial communities between agricultural fields with a history of either organic or conventional farming, and to separate the effects of soil microbial activity from the effects of other soil properties that could affect the processes under study. In two greenhouse experiments, the functional role of field-specific microbial communities was investigated for two ecosystem processes: phosphorous leaching (after artificial rainfall) and plant biomass production (using Zea mays as test plant). The soils were collected from agricultural fields on sandy soils which were managed in a conventional or organic way, pairwise co-occurring for each management type and distributed over five regions in The Netherlands. Living soil from the different fields was added to a larger fraction of sterilized soil (composed from a mixture of soil from an organically managed field and nutrient poor sand), the latter being uniform over all treatments. A similar approach of soil inoculations into a background of homogenized sterilized soil is often used in plantsoil feedback studies which aim to separate impacts of soil biota on plant growth from potential differences in abiotic soil properties (Kulmatiski and Kardol 2008; Brinkman et al. 2010). 
Intrinsically, by applying an inoculation approach as in Verbruggen et al. (2011), it is not possible to really isolate the functioning of the soil microbial community and its effects on plant performance from the abiotic soil properties, as they function within the abiotic setting provided. In the field however, soil biota modify their abiotic environment, both in physical and chemical sense, at various scales in space and time (Wright and Upadhyaya 1998; Barrios 2007). Levels of plant available soil nutrients and soil structure for instance are genuine components of soil microbial effects, which are however - intentionallynot studied in inoculation experiments (i.e. the aim is to limit abiotic carry-over effects from the inoculum soil to the soil mixture used in the experiment). Moreover, the functional significance of the rhizosphere community, including AM fungi, can be dependent on abiotic factors in the soil, and can therefore-in principle - only be properly assessed within this abiotic context. Apart from these limitations, inoculation experiments are certainly a good way to separate effects of soil microbes from the abiotic context they are operating in, potentially giving valuable insights in the role these microbes play in their original soil environment. Within this original soil environment, coupling soil processes and functions to the activity and abundance of specific belowground organisms or taxa is difficult, one reason being the complex belowground multitrophic network all soil organism are embedded in (Wardle 2006). Indeed, many biologically driven soil processes are the result of the combined activity of functionally different organisms across the different trophic levels (Wurst et al. 2012). To what extent the functional significance of one organism group in the soil-plant system can be understood by the study of individual effects, without considering multitrophic interactions, is likely dependent on the effect size of the organisms of interest and the degree of interaction with other groups of belowground biota (Wurst et al. in press, Ladygina et al. 2010). This complexity of the belowground ecosystem also implies that observational field studies involving soil microbes and their effect on soil functioning and plant growth cannot provide solid mechanistic insight in the processes at play. Consequently, controlled experiments are a requisite in elucidating the mechanisms behind soil processes in the field. Nevertheless, a combination of highly controlled (e.g. an inoculation experiment with only one microbial group in a sterile background soil) and more natural experiments (e.g. manipulating AMF abundance in the field using selective fungicide treatments) should allow for a more complete understanding of the functioning of a specific group of organisms. Experimentally gained information on individual interactions (for example an AMFplant interaction in sterile soil) can be integrated with information, obtained from field experiments and observational studies, on possible biological and abiotic factors influencing the individual interaction (for instance the presence of interacting biota and nutrient levels in the soil), and thus the functional significance of the interaction of interest in the original soil environment. This crosstalk of results from field studies and controlled experiments also guides the design of more complex controlled experiments, where additional factors (e.g. nutrient status and/or increasing complexity of soil communities) can be included.

For the specific goal of investigating which biological agents cause variation in soil functioning and ecosystem services between agricultural fields of different management, it is, as explained above, not possible to rule out the effects of long term lecagies of the microbial community on abiotic and biological characteristics of the soil, limiting the possibility to draw causal linkages in a biological manipulation experiment. For instance, a fungicide treatment applied in the field will not exclude the long term effects of fungi on for example soil structure. In this particular case, the problem is that indirect fungal effects on plant performance we may want to exclude (i.e. those that operate on long timescales and are not the effect of direct plant-fungus interactions) are still present in the manipulated system. On the other hand, the causal linkages between microbial community composition, soil functioning and plant performance which can be investigated properly in controlled inoculation experiments only comprise the short term effects of the microbial community, because the longterm effects (e.g. on soil structure, organic matter content, water retention capability) are wiped away during the experimental set up of the inoculation experiment.

Specifically for AM fungi, the experimental procedures of an inoculation study - notably the sieving and mixing of soils - can also fundamentally change their functioning by destructing mycelial networks and promoting fast over slow growing AMF species (Evans and Miller 1990, Helgason et al. 1998). Put short, it becomes clear that essential trade-offs exist 
when choosing between different approaches to study the functioning of microbes (or other soil organisms) in an ecosystem context.

An essential point we want to make is that by combining different experimental methods and observational studies, a mechanistic explanation of the effects in a simplified experimental design can be tested for consistency in field situations, where many factors and interactions occur simultaneously. Experimentally clear-cut results might be hard to translate to patterns observed in the field (e.g. Vandegehuchte et al. 2010). If such an inconsistency of experimental and field data is found, hypotheses can be formulated on why this might be so-which factors are we missing out, and which biological interactions might be in play in the field, not included in the experimental treatments?

Hopefully, the above made clear that inoculation experiments face many limitations in assessing microbial community effects on the functioning of soils. However, they can considerably add to our understanding of soil biology and associated ecosystem services. To gain insight in the mechanistic basis of soil microbial effects on ecosystem functioning using inoculation approaches, and to assess the possible discrepancies between controlled experiments and field conditions, we advocate that the following questions need to be taken in consideration.

1. Which members of the microbial community are likely to be the causing agents for the observed effects, and how can we validate this causality?

2. To what extent does the microbial community established under experimental conditions reflect the original field community of interest? This is an essential point when extrapolating experimental results to field conditions.

3. Appreciating the complex nature of plant-soil biological interactions and their soil abiotic context dependency, can we expect the functional significance one ascribes to (members of) the microbial community to be the same in the original field where the experimental inoculum originated from?

4. Given the limited (short term) timescale of most inoculation experiments, which long term effects of the microbial community on soil functioning and plant performance are possibly missed out?
Dealing with this problem is a matter of trying to integrate short term effects with long term effects, which will need to be assessed in separate studies, for example a mesocosm inoculation study and a long-term inoculation experiment in the field.

We explore the results presented in Verbruggen et al. (2011) with the above three questions in mind. In their first experiment, Verbruggen et al. (2011) found that maize plants reach significantly lower biomass in soil inoculated with live soil from the fields under study compared to a sterile control soil. However, no significant difference in plant biomass was found between organic versus conventional inoculum origin, while plant biomass was clearly more variable in the conventional inoculum treatment compared to the organic inoculum treatment. These results suggest a net negative impact of soil biota on plant growth irrespective of field management type, but more predictable 'black box' outcomes with soil biota originating from organic fields.

It is more rule than exception that plants grow better in sterile soil than on living soil (Kulmatiski et al. 2008). In other inoculation experiments, this phenomenon has been attributed to antagonistic organisms in the root environment, in many cases overruling positive plant-soil biological interactions. So who is causing the trouble for the maize in this experiment? Two observations support the interesting conclusion that AM fungi might be an important factor in this experiment, negatively affecting plant growth. First, in a soil treatment where AMF spores of Glomus intraradices were added to the sterile background soil, maize growth was significantly reduced, suggesting more costs than benefits of this plant-symbiont association under the experimental conditions (Johnson et al. 1997). Second, the extent of AMF colonization in the roots was negatively correlated with plant biomass across all the treatmentsincluding a sterile control, an AMF addition treatment and the live inocula originating from the different fields. This negative correlation between AMF colonization and plant biomass was retained when the sterilized treatment was removed from the analysis. Clearly, these observations are no proof for a causal relationship between AMF colonization extent and growth reduction of the maize plant. As the authors point out, other soil organisms could have been responsible for a direct negative effect. The strong negative correlation between 
intra-radical AMF abundance and plant biomass, together with the observation that a common AMF species reduced maize growth, make it however likely that indeed at least a part of the variation in plant growth in the experiment was attributable to plant-AMF interactions. It has to be noted that maize growers are not necessarily interested in aboveground biomass but rather in grain yield and its quality. Clearly, measuring such responses will require longer-term experiments.

In their second experiment, the authors further explored effects of the AMF communities on plant growth and phosphorous retention in the soil. Before harvesting the first experiment, watering was ceased for four weeks to promote sporulation of AM fungi. A selection of six soils from the first experiment, representing the full spectrum of AMF colonization variation, was used as an inoculum source for the second experiment. Treatments included $0 \%$ inoculum (sterile control), $4 \%$ and $12 \%$ inoculum (percentages are dry weight fraction). In order to standardize the microbial community composition across all the AMF treatments, a microbial wash composed of an AMFfree mixed filtrate from all inocula was added to all treatments.

In this second inoculation experiment, Verbruggen et al. (2011) found significantly lower intra- and extra-radical AMF colonization levels in the treatments with inoculum originating from conventional fields compared to those from organic fields. This lower AMF abundance in both the roots and in the soil was associated with higher plant biomass production, but traded-off with higher soil P-leaching. Although amounts of P leaching were not consistently related to the origin of the inocula, $\mathrm{P}$ leaching was significantly negatively correlated to AMF hyphal density in soil. Interestingly, molecular analysis revealed AMF species-specificity for both effects on plant growth and phosphorous retention. However, in response to question 2 (how well do the soil biota from controlled experiments represent the natural field communities?), the authors found that compositional divergence from the original fields had clearly occurred. AMF species richness was reduced to roughly half of the observed AMF richness in the fields. In how far other AMF properties such as total AMF abundance and community composition was affected by the experimental treatments was not further explored.Yet maize roots collected from the field did not show significant differences in \% AMF colonization between conventional and organically managed fields (Table 1 in Verbruggen et al. 2011), while in the pot experiments using soil as inoculum, colonization levels did differ between both management types (marginally non-significant higher colonization in the organic soils in experiment 1 , significantly higher colonization in the organic soils in experiment 2). This discrepancy could potentially be due to the overruling impact of disturbance on AM fungi hyphal growth in the field and greater possibility for diverging AMF growth in a pre-culturing step. Under field conditions, hyphal networks of AMF will develop, but especially physical disturbance such as soil ploughing are very destructive to these mycorrhizal structures (Helgason et al. 1998). In the study system of Verbruggen et al. (2011), the organic and conventionally managed sites differed in the application of mineral fertilisers and pesticides and in crop rotation, but tilling practices were not different (they were not mentioned as being so) and the effect of tilling may overrule effects of pesticide and fertiliser use. Comparisons between fields with different levels of physical disturbance may thus yield greater differences in colonisation levels. When using soil inoculum, AM fungi need to establish a network from germinating spores and viable AMF remains in root fragments. The use of soil inoculum after a preculturing step in the greenhouse rather than directly from the field can have promoted larger AMF densities after the pre-culturing step with soil inoculum from organic fields, given their larger diversity and assuming niche complementarity between these AMF species (van der Heijden et al. 1998).

Ultimately, the authors aimed to improve our understanding of microbial soil functioning under contrasting management types. Soil processes and the associated ecosystem services are the result of a complex interplay of abiotic soil factors and biological activity in the rhizosphere and bulk soil. Communities of AMF might have different functional roles in soils of different management type due to differences in their abundance and composition (Oehl et al. 2003; Verbruggen et al. 2010) and/or due to the different abiotic and biotic context in which they need to function. From an agro-ecological perspective, this means that the net effect of AMF on the studied processes might be quite different in conventional versus organically managed systems. In conventional farming, AMF 
might only imply costs for the plant, because mineral phosphorous and other nutrients are generally added to the fields. In an organic farming context, AMF might be essential to prevent $\mathrm{P}$ limitation in the long run. This means that the functional significance of AMF can indeed be dependent on management type. One could further speculate about important interactions between AMF and other soil organisms, changing AMF community composition during the experimental treatments, and so on - all of this possibly dragging the obtained results out of a proper field context.

The considerations discussed above do however not preclude the quality and importance of the paper of Verbruggen et al. (2011). The study provides very interesting perspectives on the multi-functional role of AM fungi in agricultural systems and potential tradeoffs between several functions provided by AMF. However, we believe that firm conclusions on the actual functional outcomes of AMF communities from agricultural systems with either conventional or organic management are hard to make on the basis of the results presented in Verbruggen et al. (2011). The potential trade-off between nutrient retention and plant productivity in the AMF-maize interaction might hold true on a mechanistic basis, meaning that these individual effects of AMF (carbon cost for the plant and positive effect on $\mathrm{P}$ retention) potentially exist in field situations. The ultimate question for farming policy, however, is if this trade-off holds true in the full context of field conditions and in a wide range of soil types and levels of soil nutrients. Organic farming relies on internal, biologically driven nutrient cycling by retaining nutrients in the soil system in the form of organic matter and mineralization of internal and externally provided organic matter by soil biota (Altieri 1999). AMF communities play an important role in these processes - as the study of Verbruggen et al. (2011) confirms (for P retention). Loss of AMF diversity or activity, especially of those adapted to the local abiotic and biotic environment in which they function, might thus negatively affect both $\mathrm{P}$ retention and crop production in the long run. Moreover, AMF are known to provide other ecosystem functions beyond $\mathrm{P}$ retention, such as suppression of soil pathogens and improving soil structure, which need to be taken into account in order to make up the balance between costs and benefits of AMF mediated impact of agricultural practices (Hart and Trevors 2005).
We conclude that Verbruggen et al. (2011) make an interesting contribution to our understanding of the functional role of AMF communities for the studied processes, but that the results of this paper only allow us to merely speculate about the realized functional role of the management-specific AMF communities in the complex web of below ground biological interactions and the complexity of the abiotic soil environment. We recommend that highly controlled experiments, such as the one discussed in this commentary, be complemented by long-term field studies where short as well as longer-term impacts of (manipulated) soil biota communities can be investigated, ideally across soils with different abiotic properties. As an intermediate between field and highly controlled mesocosm studies with inoculation of single (trophic) groups of soil biota, mesocosm studies with increasing biotic complexity and in different abiotic settings (e.g. nutrient levels) could provide an additional stepping stone in increasing our mechanistic understanding of the functioning of specific soil biota in their natural complex biotic and abiotic environment.

Acknowledgements We would like to thank Pella Brinkman for critical comments on a first version of the paper. We thank the authors of Verbruggen et al. (2011) (this volume) for their study, providing us with food for thought for this commentary. GBDD was supported by a Marie Curie Intra European Fellowship within the 7th European Community Framework Programme. NIOO publication 5087.

Open Access This article is distributed under the terms of the Creative Commons Attribution Noncommercial License which permits any noncommercial use, distribution, and reproduction in any medium, provided the original author(s) and source are credited.

\section{References}

Altieri MA (1999) The ecological role of biodiversity in agroecosystems. Agric Ecosyst Environ 74:19-31

Azcon-Aguilar C, Barea JM (1996) Arbuscular mycorrhizas and biological control of soil-borne plant pathogensAn overview of the mechanisms involved. Mycorrhiza 6:457-464

Barea JM, Azcón-Aguilar C, Azcón R (1996) Interactions between mycorrhizal fungi and rhizophere microorganisms within the context of sustainable soil-plant systems. In: Gange AC, Brown VK (eds) Multitrophic interactions in terrestrial systems. Blackwell, Oxford, pp 65-77

Barrios E (2007) Soil biota, ecosystem services and land productivity. Ecol Econ 64:269-285 
Brinkman EP, Van der Putten WH, Bakker EJ, Verhoeven KJF (2010) Plant-soil feedback: experimental approaches, statistical analyses and ecological interpretations. J Ecol 98:1063-1073

Bronick CJ, Lal R (2005) Soil structure and management: a review. Geoderma 124:3-22

Brussaard L, Behan-Pelletier VM, Bignell DE, Brown VK, Didden W, Folgarait P, Fragoso C, Freckman DW, Gupta V, Hattori T, Hawksworth DL, Klopatek C, Lavelle P, Malloch DW, Rusek J, Soderstrom B, Tiedje JM, Virginia RA (1997) Biodiversity and ecosystem functioning in soil. Ambio 26:563-570

Buée M, De Boer W, Martin F, van Overbeek L, Jurkevitch E (2009) The rhizosphere zoo: an overview of plantassociated communities of microorganisms, including phages, bacteria, archaea, and fungi, and of some of their structuring factors. Plant Soil 321:189-212

Coleman DC, Crossley DA Jr, Hendrix PF (2004) Fundamentals of Soil Ecology, 2nd edn. Elsevier, Amsterdam

De Deyn GB, Raaijmakers CE, Van der Putten WH (2004) Plant community development is affected by nutrients and soil biota. J Ecol 92:824-834

De Deyn GB, Cornelissen JHC, Bardgett RD (2008) Plant functional traits and soil carbon sequestration in contrasting biomes. Ecol Lett 11:516-531

Evans DG, Miller MH (1990) The role of the external mycelial network in the effect of soil disturbance upon vesiculararbuscular mycorrhizal colonization of maize. New Phytol 114:65-71

Garbeva P, van Veen JA, van Elsas JD (2004) Microbial diversity in soil: selection of microbial populations by plant and soil type and implications for disease suppressiveness. Annu Rev Phytopathol 42:243-270

Gomiero T, Pimentel D, Paoletti MG (2011) Is there a need for a more sustainable agriculture? Crit Rev plant Sci 30SI:6-23

Hart MM, Trevors JT (2005) Microbe management: application of mycorrhyzal fungi in sustainable agriculture. Front Ecol Environ 3:533-539

Helgason T, Daniell TJ, Husband R, Fitter AH, Young JPW (1998) Ploughing up the wood-wide web? Nature 394:431-431

Hoeksema JD, Chaudhary VB, Gehring CA, Johnson NC, Karst J, Koide RT, Pringle A, Zabinski C, Bever JD, Moore JC, Wilson GWT, Klironomos JN, Umbanhowar J (2010) A meta-analysis of context-dependency in plant response to inoculation with mycorrhizal fungi. Ecol Lett 13:394-407

Jackson AO, Taylor CB (1996) Plant-microbe interactions: life and death at the interface. Plant Cell 8:1651-1668

Johnson NC, Graham JH, Smith FA (1997) Functioning of mycorrhizal associations along the mutualism-parasitism continuum. New Phytol 135:575-586

Klironomos JN (2003) Variation in plant response to native and exotic arbuscular mycorrhizal fungi. Ecology $84: 2292-2301$
Kulmatiski A, Kardol P (2008) Getting plant-soil feedbacks out of the greenhouse: experimental and conceptual approaches. In: Luttge UE, Esser K, Beyschlag W, Murata J (eds) Progress in Botany-69 (Review Genetic Physiology Ecology). Springer, Berlin Heidelberg, pp 449-472

Kulmatiski A, Beard KH, Stevens JR, Cobbold SM (2008) Plant-soil feedbacks: a meta-analytical review. Ecol Lett 11:980-992

Kuyper TW, Giller KE (2011) Biodiversity and ecosystem functioning below-ground. In: Lenné JM (ed) Agrobiodiversity Management for Food Security - a critical review. CABI, Oxfordshire, pp 134-149

Ladygina N, Henry F, Kant MR, Koller R, Reidinger S, Rodriguez A, Saj S, Sonnemann I, Witt C, Wurst S (2010) Additive and interactive effects of functionally dissimilar soil organisms on a grassland plant community. Soil boil biochem 42:2266-2275

Oehl F, Sieverding E, Ineichen K, Mader P, Boller T, Wiemken A (2003) Impact of land use intensity on the species diversity of arbuscular mycorrhizal fungi in agroecosystems of Central Europe. App Environ Microb 69:2816-2824

van der Heijden MGA, Klironomos JN, Ursic M, Moutoglis P, Streitwolf-Engel R, Boller T, Wiemken A, Sanders IR (1998) Mycorrhizal fungal diversity determines plant biodiversity, ecosystem variability and productivity. Nature 396:69-72

Vandegehuchte ML, de la Peña E, Bonte D (2010) Interactions between root and shoot herbivores of Ammophila arenaria in the laboratory do not translate into correlated abundances in the field. Oikos 119:1011-1019

Vazquez MM, Cesar S, Azcon R, Barea JM (2000) Interactions between arbuscular mycorrhizal fungi and other microbial inoculants (Azospirillum, Pseudomonas, Trichoderma) and their effects on microbial population and enzyme activities in the rhizosphere of maize plants. Appl Soil Ecol 15:261-272

Verbruggen E, Roling WFM, Gamper HA, Kowalchuk GA, Verhoef HA, van der Heijden MGA (2010) Positive effects of organic farming on below-ground mutualists: largescale comparison of mycorrhizal fungal communities in agricultural soils. New Phytol 186:968-979

Verbruggen E, Kiers ET, Bakelaar PNC, Röling WFM, van der Heijden MGA (2011) Provision of contrasting ecosystem services 1 by soil communities from different agricultural fields. Plant and Soil XXX: XXX-XXX

Wardle DA (2006) The influence of biotic interactions on soil biodiversity. Ecol Lett 9:870-556

Wright SF, Upadhyaya A (1998) A survey of soils for aggregate stability and glomalin, a glycoprotein produced by hyphae of arbuscular mycorrhizal fungi. Plant Soil 198:97-107

Wurst S, Orwin KA, De Deyn GB (2012) Soil Biodiversity and Functions. In: Wall DH (ed) Oxford Handbook of Soil Ecology and Ecosystem Services. Oxford University Press, Oxford, pp XXX-XXX 